\title{
Gut microbial dysbiosis as a limiting factor in the management of primary and secondary sarcopenia: an Asian Indian perspective
}

Citation for published version (APA):

Bhattacharya, S., Bhadra, R., Schols, A. M. W. J., \& Sambashivaiah, S. (2020). Gut microbial dysbiosis as a limiting factor in the management of primary and secondary sarcopenia: an Asian Indian perspective. Current Opinion in Clinical Nutrition and Metabolic Care, 23(6), 404-410. https://doi.org/10.1097/MCO.0000000000000688

Document status and date:

Published: 01/11/2020

DOI:

10.1097/MCO.0000000000000688

Document Version:

Publisher's PDF, also known as Version of record

\section{Document license:}

Taverne

Please check the document version of this publication:

- A submitted manuscript is the version of the article upon submission and before peer-review. There can be important differences between the submitted version and the official published version of record.

People interested in the research are advised to contact the author for the final version of the publication, or visit the DOI to the publisher's website.

- The final author version and the galley proof are versions of the publication after peer review.

- The final published version features the final layout of the paper including the volume, issue and page numbers.

Link to publication

\footnotetext{
General rights rights.

- You may freely distribute the URL identifying the publication in the public portal. please follow below link for the End User Agreement:

www.umlib.nl/taverne-license

Take down policy

If you believe that this document breaches copyright please contact us at:

repository@maastrichtuniversity.nl

providing details and we will investigate your claim.
}

Copyright and moral rights for the publications made accessible in the public portal are retained by the authors and/or other copyright owners and it is a condition of accessing publications that users recognise and abide by the legal requirements associated with these

- Users may download and print one copy of any publication from the public portal for the purpose of private study or research.

- You may not further distribute the material or use it for any profit-making activity or commercial gain

If the publication is distributed under the terms of Article $25 \mathrm{fa}$ of the Dutch Copyright Act, indicated by the "Taverne" license above, 


\title{
Gut microbial dysbiosis as a limiting factor in the management of primary and secondary sarcopenia: an Asian Indian perspective
}

\author{
Shinjini Bhattacharya ${ }^{\mathrm{a}, *}$, Rohini Bhadra ${ }^{\mathrm{a}, *}$, Annemie M.W.J. Schols ${ }^{\mathrm{b}}$, \\ and Sucharita Sambashivaiah ${ }^{\mathrm{C}}$
}

\begin{abstract}
Purpose of review
The article summarizes recent research advances on the role of gut microbiome in primary and secondary sarcopenia. This article also explores the potential contribution of gut dysbiosis to suboptimal sarcopenia management with special focus on factors contributing to gut dysbiosis among Asian Indians.
\end{abstract}

\begin{abstract}
Recent findings
Aging and chronic diseases contribute to gut dysbiosis and intestinal barrier dysfunction allowing enhanced microbial translocation that may negatively affect muscle strength, physical function, and frailty. Gut microbiome of Asian Indians has shown a unique composition that is affected by multiple factors, such as socioeconomic status, poor hygiene, high rate of infection and infestations, antibiotic overuse and transition towards a westernized eating pattern. Current management approach for sarcopenia (exercise and/or protein supplementation) fails to address gut dysbiosis and intestinal barrier dysfunction. Incorporating a prebiotic or probiotic element to the intervention strategy may improve gut dysbiosis, inflammation and muscle function.
\end{abstract}

\section{Summary}

Gut dysbiosis and intestinal barrier dysfunction appear to be a significant limitation in sarcopenia management, thus gut centric intervention may be perceived as a (co)intervention strategy to be tested in appropriate clinical trials.

\section{Keywords}

aging, gut microbiome, gut-centric intervention, muscle strength, sanitation

\section{INTRODUCTION}

Sarcopenia is characterized by the progressive loss of skeletal muscle mass and function with advancing age. It is one of the major causes of age-associated frailty, disability and mortality. Prevalence of sarcopenia varies greatly among various populations around the world [1]. Its prevalence in India varies from 1.6 to $36.6 \%$ among different communities [2]. Sarcopenia is of two types, distinguishable based on its chief etiology. Primary sarcopenia is mainly agerelated with no other cause, whereas secondary sarcopenia is associated with factors other than (or in addition to) aging, such as malnutrition, reduced physical activity and chronic disease [3]. Several mechanisms may contribute to muscle loss, including decreased protein synthesis or anabolic resistance, increased protein breakdown or impaired muscle regeneration because of multiple causes including chronic low-grade inflammation, oxidative stress and mitochondrial damage $[4,5]$. The effect of these factors may be enhanced when combined with physical inactivity, malnutrition and the presence of one or more chronic diseases, all commonly prevailing in the elderly population. Gut dysbiosis and associated inflammation have been speculated as being a contributory factor in the development of sarcopenia. Gut dysbiosis is defined as the presence of

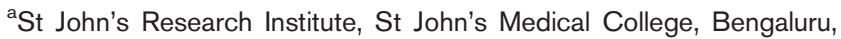
India, 'NUTRIM School of Nutrition and Translational Research in Metabolism, Department of Respiratory Medicine, Maastricht University, The Netherlands and 'Department of Physiology, St John's Medical College, Bengaluru, India

Correspondence to Sucharita Sambashivaiah, MD, PhD, Professor, Department of Physiology, St John's Medical College, Bengaluru 560034, India. Tel: +91 80 49466324; e-mail: sucharita@stjohns.in

*Shinjini Bhattacharya and Rohini Bhadra have equally contributed and share joint first authorship.
}

Curr Opin Clin Nutr Metab Care 2020, 23:404-410

DOI:10.1097/MCO.0000000000000688 


\section{KEY POINTS}

- Gut dysbiosis and altered intestinal membrane permeability may accelerate the process of sarcopenia associated with aging and chronic diseases.

- Multiple environmental factors are responsible for negative modulation of gut microbiome in Asian Indians, which includes poor sanitation, increased infection, infestation, antibiotic overuse and dietary variation.

- Gut dysbiosis may be recognized as the potential contributor to sub-optimal management of sarcopenia.

- Gut microbiome represents a promising intervention site for management of sarcopenia.

an imbalanced, low-diversity, intestinal microbiota [6]. The microbiota of the elderly, in general, shows decreased diversity [6]. The association between gut microbiota alteration and modulation of whole-body lean mass was first established in a pioneering animal study by Bakhed et al. [7]. A decrease in whole body lean mass and increase in fat mass was observed in germ-free mice following colonization with fecal samples of conventionally raised mice [7], indicating sarcopenia and sarcopenic obesity. Increasing literature evidence identifies the gut microbial composition as a key contributor to skeletal muscle mass, metabolism and function during aging, and thus might be an important determinant in development of sarcopenia [5], giving rise to the concept of the gutmuscle axis. Various studies have indeed shown that increase in microbial production of short-chain fatty acids, such as butyrate, is a potential modulator of muscle strength and physical function in humans $\left[8^{-"}, 9\right]$. The gut-muscle axis has also become an area of interest for researchers focusing on the development and management of sarcopenia. In the past year, several studies have been conducted in this area, worldwide. Remarkably, in India, direct studies have been scarce although there is evidence of various factors affecting gut health among Indians, which might be linked to muscle health as well. This review will, therefore, summarize the recent literature evidence on the above-mentioned areas to explore the contribution of gut dysbiosis to suboptimal sarcopenia management, with a specific focus on factors that might be contributory to gut dysbiosis among Asian Indians.

\section{AGING GUT AND SARCOPENIA}

Gut microbiota has been causally linked to systemic low-grade inflammation that is observed in both aging and chronic disease. Enhanced production of pro-inflammatory cytokines, such as IL-6, TNF$\alpha$ and IL- $1 \beta$ by intestinal epithelial cells is increased with advanced age. This, in turn, could cause increased intestinal epithelial tight junction permeability giving rise to intestinal barrier dysfunction. Indeed, a study by Sovran et al. [10"] has reported a reduction (or even complete absence) in colon mucus layer of older mice (19 months) than younger controls (10 months), thus indicating a decreased intestinal barrier function with aging.

The gastrointestinal tract acts as the major barrier in the translocation of microbial products in both animals and humans. The barrier is composed of various physical, biochemical, immunological and microbial elements. Defects in these barrier elements, a phenomenon common in aging, can result in an elevated immune reaction and inflammation by causing an increased translocation of microbial products into the systemic circulation. This barrier dysfunction-induced systemic inflammation might have significant clinical implications in the form of metabolic syndrome, decreased muscle strength and physical function [11"]. This is supported by the observation of Stehle et al. [11"] where a significant decrease in physical function and grip strength was reported among healthy older adults with a simultaneous increase in plasma levels of lipopolysaccharide binding protein (LBP), as a biomarker of microbial translocation and hence, intestinal barrier dysfunction. Ambulatory $(<60 \mathrm{~min} /$ week moderate structured physical activity) community dwelling elderly individuals of 6079 years of age, having a BMI between 28 and $40 \mathrm{~kg} /$ $\mathrm{m}^{2}$ with either cardiovascular disease or cardiometabolic dysfunction and a self-reported limitation in mobility, demonstrated a significantly strong association between baseline markers of microbial translocation and inflammatory markers. The microbial markers measured by LBP- 1 and sCD-14 levels were strongly associated with IL-6 and IL-8. Furthermore, a negative association between LBP-1 levels and physical function [assessed by the short physical performance battery (SPPB) and $400 \mathrm{~m}$ walk test] was found in the above-mentioned study group [11"]. Lifestyle interventions (diet and exercise) were shown to have no effect on intestinal barrier function and inflammation despite successful weight reduction among the study group [11"]. This suggests that diet and exercise are possibly not universally beneficial for restoring intestinal barrier competency, and hence alternative or additional strategies might need to be explored for reducing microbial translocation and inflammatory burden in older adults. Other studies conducted previously have also shown chronic inflammation (measured 
by IL-6, TNF- $\alpha$ and CRP levels) elicited by microbial translocation to be negatively associated with physical function (measured by SPPB, $4 \mathrm{~m}$ walk and repeated chair stands) in older adults with multiple comorbidities [11"].

Decreased intestinal barrier function was shown by Sovran et al. [10"]. Evidence of altered fecal microbial composition and expression of immunity in intestinal mucosal tissue with aging was demonstrated [10"]. The gut-based study among centenarian has found high proteobacteria, a pathobiont (pathogenic in certain circumstances), shown to cause negative health effects in susceptible hosts [20]. Therefore, maintaining gastrointestinal barrier integrity is important for the microbial homeostasis.

Association of gut microbial profile with physical frailty and sarcopenia was shown in a study by Picca et al. where microbial alpha diversity (the variance within the sample) between sarcopenic and nonsarcopenic groups did not show any significant difference, however, analysis of differential abundance of microbial taxa showed increased Peptostreptococcaceae and Bifidobacteriaceae at the family level and depletion of Slackia and Eubacterium in participants with sarcopenia. The taxa linked to sarcopenia in this study were also associated with biological aging and frailty in other studies conducted previously [8"'].

An overall shift towards butyrate-producing bacteria, which may be comparable to that observed in higher functioning people was observed in this study [8"']. Butyrate, itself, has been shown to have a beneficial effect on the intestinal barrier function by reinforcing intestinal epithelial tight junction and preventing microbial translocation and reducing systemic inflammation, as well as, improving muscle bioenergetics and restricting myosteatosis by promoting fatty acid oxidation. This characteristic of butyrate shift among frail individuals may suggest a positive role of butyrate-producing microbes in muscle function [8"']. One possible reasoning for this may be a compensatory mechanism to counteract the loss of muscle mass, strength and function associated with sarcopenia but this warrants further investigation for confirmation.

Reduced SCFA production may also trigger insulin resistance and result in increased fatty acid deposition within the muscle, lowering muscle quality, thus having an indirect effect on muscle quality. The ensuing lower muscle quality may further promote insulin resistance, feeding a vicious circle that contributes to the onset and progression of sarcopenia, frailty and possibly even sarcopenic obesity $\left[8^{-"}\right]$.

\section{GUT DYSBIOSIS ASSOCIATED WITH}

\section{CHRONIC DISEASES AND SARCOPENIA}

Chronic organ dysfunction including liver disease, chronic kidney disease (CKD) and chronic obstructive pulmonary disease (COPD) may contribute or even accelerate the development of sarcopenia. Gut dysbiosis and systemic inflammation have also been a consistent finding in many of these conditions that impair muscle functionality.

Altered gut microbiome composition has been reported in predialysis CKD patients with a higher relative abundance of genera Lactobacillus, Coprobacillus, Anaerotruncus and Citrobacter species and lower Prevotella, F. prausnitzii and Roseburia (the saccharolytic and butyrate-producing species). This alteration in gut microbiota was further associated with frailty most likely mediated through production of toxic bacterial metabolites [12].

Similarly, patients with liver disease have shown marked alteration in the gut microbiome and/or inflammatory profile. Gut microbiome analysis of nonalcoholic steatohepatitis (NASH) patients revealed an abundance of Escherichia coli [13]. It is noteworthy, that the abundance of genus Escherichia is associated with production of alcohol that induces inflammation. A recent publication used $E$. coli lipopolysaccharide for immune system stimulation in a porcine model and reported a decrease in whole-body protein synthesis, a reduced muscle fiber cross-sectional area and a switch in muscle fibers towards a slow-twitch oxidative type (type I) with lower fast-twitching glycolytic fibers (type II) [14"']; all the observations indicative of muscle atrophy. Furthermore, it has been reported that chronic alcoholics having significantly lower mean handgrip strength than controls reported to have gut microbiome profile skewed towards pro-inflammatory direction [15]. Surprisingly, incorporating gutbased intervention, that is, multistrain probiotic supplementation in dynapenic (loss of muscle strength) cirrhosis patient failed to demonstrate significant improvement in muscle strength after 12-week probiotic supplementation, despite improvement in intestinal barrier function and inflammatory response [16]. However, the study did not include objective measures of muscle mass. Furthermore, the study lacked exercise or nutrition support, considered to be crucial for the improvement in muscle quality. Thus, the study outcome might not reflect the true impact of gut-based (co)interventions on muscle health.

Increased small intestinal permeability has been reported in COPD patients during acute exacerbations as reflected by an increased inflammatory response and higher urinary lactulose/L-rhamnose ratio during hospitalization [17]. The underlying 
mechanisms are not yet known, however, authors speculated that inflammation and hypoxic damage as the cause of intestinal barrier disruption. The contribution of altered gut microbiome being a strong predictor of intestinal barrier integrity requires further investigation. The available evidences on COPD patients including experimental COPD models are pointing towards an accelerated and stepwise decline in the muscle mass, which is suggested to be related to the frequency of acute exacerbations [18].

Studies conducted on patients of metabolic syndrome have shown the beneficial effect of probiotic supplementation on the improvement in inflammatory markers and insulin resistance. However, the direct impact of the changes in gut microbiome on muscle health was not examined $[19,20]$. Therefore, this might be a potential pathway to explore for exhibiting the favorable effect of gut microbiota on muscle functionality in metabolic syndrome or diabetes.

The recent evidences point towards the altered gut microbial profile in chronic organ dysfunction. There is a decrease in commensal microbes, abundance of pathogenic bacteria, increase in intestinal permeability and inflammatory response. These factors can alter the muscle physiological and biochemical properties in a catabolic direction thereby accelerating the development and progression of sarcopenia.

\section{GUT MICROBIAL PROFILE IN INDIANS}

Gut microbiome metagenomic studies have illustrated that there are population-specific signatures in microbial composition. Studies on gut microbiome of adult Indians with no comorbidity have shown a distinctive composition with a predominance of Prevotella as the most discriminatory genus $\left[21^{*}, 22,23\right]$. Prevotella belongs to phylum Bacteroidetes typically associated with production of SCFA. However, literature suggests, among Bacteroidetes, Prevotella shows the lowest genetic potential of producing SCFA. Further it is frequently linked with chronic inflammation [21"]. Gut microbiome composition of rural Indians have demonstrated a difference in bacterial abundance with age. A higher abundance of Ruminococcus has been reported in healthy older adults (age $\geq 50$ years) [23]. Gut microbiome analysis of Indian centenarians has shown a higher species richness and biodiversity of family Ruminococcaceae, accompanied with a decrease in Prevotella species [24]. These findings are indicative of the beneficial role of Ruminococcaceae to facilitate healthy aging, also indicating the negative association of Prevotella with longevity and well being. It is noteworthy that the members of Ruminococcaceae family were reported to be a major butyrate producer, which is indicative of its potential role in preventing inflammation and immunosenescence [24]. These evidences primarily concentrate on gut microbial profile of healthy Indians while the changes with aging, chronic disease or sarcopenia is still lacking. Therefore, it is difficult to conclude the discriminative alterations in gut microbiome of elderly Indians with sarcopenia or chronic disease.

Although there is paucity in literature linking aging, chronic disease and the gut-muscle axis published in the past year for the Indian population, we have come across several population-specific causes of gut dysbiosis in Indians, which might have a subsequent implication on muscle health. Environmental factors, such as diet, lifestyle and hygiene have shown a strong influence on microbial composition in many of the studies that might have a profound effect on the gut-muscle axis. Identification of these factors is instrumental to establish therapeutic and preventive measures for sarcopenia.

\section{Poor sanitation, related infections and antibiotic resistance}

Over the past few decades, researchers have emphasized the 'hygiene hypothesis', which implies insufficient exposure to microbes in developed countries with high levels of hygiene may restrict the spectrum of bacterial colonization, thus reducing microbial enrichment and biodiversity. Considering this hypothesis, the population in developing countries should be expected to have better microbial composition because of increased exposure to a wide variety of microbes. However, low socioeconomic status in developing countries contributes to poor sanitation and inadequate healthcare infrastructure, which results in a prominent increase in infection rates. This leads to rampant use of antibiotics that can eliminate the sensitive bacterial strains and favor the growth of antibiotic-resistant strains thereby supporting the spread of antibiotic-resistant genes in gut microbiota. The depletion of commensal bacterial strains because of antibiotic treatment was associated with a reduction in SCFA production and increased gut inflammation in mice during oral Candida albicans infection, which was resolved following SCFA administration [25]. Furthermore, certain antibiotics, such as a combination of cefoperazone, clindamycin, and vancomycin may eliminate Lachnospiraceae and Ruminococcaceae families. Ruminococcaceae, as mentioned above is associated with healthy aging, and loss of this family is indeed detrimental to aging health. Also, both 
Lachnospiraceae and Ruminococcaceae are associated with the production of secondary bile acids in intestinal lumen that restricts the growth of pathogen Clostridium difficile, a Gram-positive anaerobe associated with diarrheal disease. Thus, loss of the above-mentioned microbial families because of antibiotic treatment may increase the risk of $C$. difficile infection [25]. A recent study on Indian population investigating the burden of antimicrobial resistance and diarrheal diseases, particularly $C$. difficile, in rural and urban population of central India, reported a higher burden of $C$. difficile in the urban and peri-urban populations with higher exposure to antibiotics, many of which carried antibiotic resistance genes to virtually every class of antibiotic [26"].

\section{Parasitic infestation}

Parasitic disease, a secondary consequence of poor sanitation, widely prevalent in developing countries, such as India, might have specific interaction with the subset of microbial population. For instance, Entamoeba histolytica, a protist parasite that causes amoebiasis, was associated with phagocytosis of beneficial bacteria. E. histolytica exhibited preferential phagocytosis to Lactobacillales, Erysipelotrichales, Clostridales, and Bifidobacteriales family, which are essential for maintaining healthy gut microbial population $\left[27^{-}\right]$. Thus, wiping out the favorable bacterial families may trigger gut dysbiosis and associated inflammatory response.

\section{Dietary habits and lifestyle}

India is experiencing a steep rise in urbanization and westernization in line with the global trend. There is a drastic increase in sedentarism in urbanized population, which is accompanied with a transition towards ready-to-eat, factory-processed eating pattern that has replaced the naturally produced unprocessed staples. This major shift in diet and lifestyle affects the intestinal microbial population to a great extent. Westernized eating pattern has been shown to shift the microbial population towards Bacteroides dominance [28], which is linked to adiposity and metabolic disease. In the Indian population, a higher abundance of taxa belonging to Bacteroidetes was reported in urban inhabitants following a western-type diet, typically high in protein and fat $\left[29^{\prime}\right]$. An overall higher gut microbial diversity has been observed in the tribal population compared with the urban [29"]. However, Kaur et al. reported a higher proportion of Bacteroidetes in Ladakh ethnic tribes and abundance of Actinobacteria and Firmicutes in Jaisalmer and Khargone participants respectively. Also, healthy population from Ladakh had alpha diversity as low as the US population [30]. It is noteworthy that the dietary pattern of Ladakh ethnic group was predominantly nonvegetarian with increased intake of fat and protein, whereas the majority of Jaisalmer participants were vegetarians and Khargone participants had a mixed eating pattern.

The challenges of exploring the gut microbial composition of Indians lie mainly in the vast diversity of the population in relation to their geographic locations, genetic make-up and dietary habits among others [31]. Environment has been shown to extensively affect the microbial population, richness and biodiversity in addition to aging and chronic disease. In a developing country like India, environmental components, such as infection, antibiotic overuse, parasites, dietary variation in line with the urbanization, appear to be the major source of gut dysbiosis. As, gut dysbiosis has the potential to cause deterioration of muscle health with aging and given the already established prevalence of sarcopenia among Indians, despite scarcity of recent literature on the Indian gut-muscle axis, therapeutic strategies targeting gut microbiome is extremely important in Asian Indians and may be indispensable to overall wellbeing.

\section{GUT-CENTRIC INTERVENTION AS A NOVEL THERAPEUTIC APPROACH TO SARCOPENIA MANAGEMIENT}

The conventional therapy for the management of sarcopenia and ensuing frailty that remains most explored includes exercise and protein supplementation [32]. Various studies have been conducted over the years with mixed results. Significant increase in handgrip strength and improvement in the physical function was observed in prefrail and frail older adults following a 12-week resistance exercise with protein supplementation compared with a control group receiving only resistance exercise (both groups received a weight maintenance diet) [32]. Assessment of the effect of a 13-week Vitamin D and leucine-enriched whey protein intervention on chronic low-grade inflammatory profile (CLIP) in sarcopenia, found an overall significant increase in CLIP, demonstrated by an increase in IL6 and IL-1RA [33].

To date, resistance exercise and high protein intake have been advocated in the management of sarcopenia. However, recent literature suggests that high protein intake itself might have a negative impact on gut microbiota by shifting the gut bacterial metabolism towards amino acid degradation and fermentation [ $\left.8^{-"}\right]$. Furthermore, age-related alteration in the gut microbial composition and associated inflammation can contribute to anabolic 


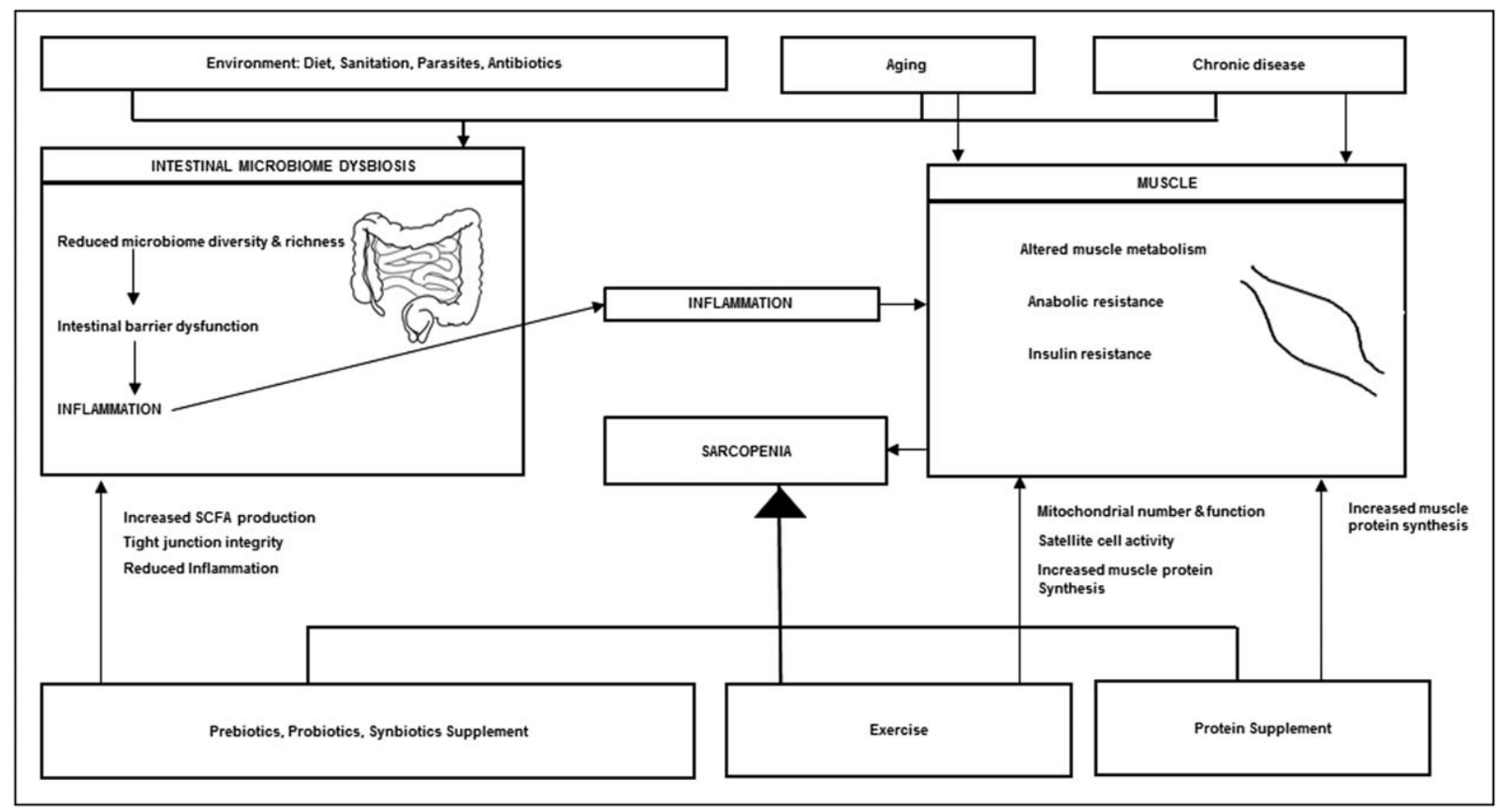

FIGURE 1. Illustration of gut-muscle axis and potential gut-based (co)intervention to ameliorate sarcopenia: aging and chronic disease are potential contributors to muscle degradation, and when accompanied with environmental factors, it mediates intestinal dysfunction inducing inflammation, a key mechanism for sarcopenia development. Hence, incorporating a gut-centric supplementation to the current management might bring about a more wholesome perspective to the management strategy by overcoming the piffalls of the current approach.

resistance as it affects the absorption and utilization of protein in small intestine that results in a higher requirement of proteins for muscle protein synthesis [4]. Therefore, the role of gut bacteria in nutrient signaling to the host might need to be monitored and applied in the nutritional management of sarcopenia.

Gut-based intervention seems to be a promising site of (co)intervention to improve muscle mass, physical performance and frailty indices as evidenced by multiple literature. Supplementation of high-dose probiotic has been associated with an increase in muscle mass and a significant improvement in exercise performance in healthy adults [34"']. Frailty index score was significantly reduced in nondemented elderly individuals on prebiotic supplementation [35]. Supplementation of heatkilled Bifidobacterium breve B3 has shown to increase the weight of the soleus muscle in mice. HK-B3 also promoted muscle mitochondrial biogenesis through the AMPK-PGC-1 signaling pathway and significantly increased expression of peroxisome proliferator-activated receptor gamma coactivator (PGC)-1 $\alpha$, which is known to shift muscle fiber distribution to a more oxidative fiber type (type II). The HK-B3 group was also characterized by increase in muscular strength measured by handgrip [36]. Supplementation of inulin or inulin propionate ester supplementation (IPE) improved markers of systemic inflammation and insulin resistance in adults between 18 and 65 years of age, both of which are detrimental to muscle quality [37]. Thus, probiotic or prebiotic supplementation appears to positively modulate age-related muscle loss and frailty, but the relative improvement compared with exercise or in combination with exercise needs further investigation.

Given the evidences to date, gut microbiota and metabolites appear to be a potential target for intervention in the context of sarcopenia and frailty. Thus, gut-centric intervention may be a novel therapeutic approach worth exploring in mitigating sarcopenia (Fig. 1).

\section{CONCLUSION}

Gut microbiota plays an active role in the development of both primary and secondary sarcopenia. Though, human trials on the gut-muscle axis is still in the early phase, there is clear evidence suggesting an association between the gut microbial composition, membrane permeability and inflammation with measures of muscle strength and physical function. The conventional method of sarcopenia management involves exercise and protein supplementation; however, exploration of the gut-centric approach could emerge as a novel intervention therapy for effective multimodal sarcopenia management. 


\section{Acknowledgements}

The authors of the review would like to acknowledge the Indian Council of Medical Research [sanction number 5/ 9/1225/2019-Nut] and DBT/Wellcome Trust India Alliance Fellowship [grant number IA/I/13/1/500907] awarded to $S S . S B$ and $R B$ are part of the above grants as research fellows.

\section{Financial support and sponsorship}

None.

\section{Conflicts of interest}

There are no conflicts of interest.

\section{REFERENCES AND RECOMIMENDED \\ READING}

Papers of particular interest, published within the annual period of review, have been highlighted as:

- of special interest

-1. of outstanding interest

1. Shafiee G, Keshtkar A, Soltani A, et al. Prevalence of sarcopenia in the world: a systematic review and meta- analysis of general population studies. J Diabetes Metab Disord 2017; 16:21.

2. Pal R, Aggarwal A, Singh $T$, et al. Diagnostic cut-offs, prevalence, and biochemical predictors of sarcopenia in healthy Indian adults: the Sarcopenia-Chandigarh Urban Bone Epidemiological Study (Sarco-CUBES) [published online ahead of print, 2020 Jun 5]. Eur Geriatr Med 2020; 10.1007/ s41999-020-00332-z.

3. Santilli V, Bernetti A, Mangone M, Paoloni M. Clinical definition of sarcopenia. Clin Cases Miner Bone Metab 2014; 11:177-180.

4. Casati M, Ferri E, Azzolino D, et al. Gut microbiota and physical frailty through the mediation of sarcopenia. Exp Gerontol 2019; 124:110639.

5. Ticinesi A, Nouvenne A, Cerundolo N, et al. Gut microbiota, muscle mass and function in aging: a focus on physical frailty and sarcopenia. Nutrients 2019; 11 :.

6. Morita $\mathrm{E}$, Yokoyama $\mathrm{H}$, Imai $\mathrm{D}$, et al. Aerobic exercise training with brisk walking increases intestinal Bacteroides in healthy elderly women. Nutrients $2019 ; 11: 868$

7. Bäckhed F, Ding H, Wang T, et al. The gut microbiota as an environmental factor that regulates fat storage. Proc Natl Acad Sci U S A 2004;101:15718-15723.

8. Picca A, Ponziani FR, Calvani R, et al. Gut microbial, inflammatory and

metabolic signatures in older people with physical frailty and sarcopenia: results from the BIOSPHERE study. Nutrients 2019; 12:65.

This study highlighted that gut microbial changes may be associated with development of sarcopenia and frailty. It also highlighted the negative aspect of conventional sarcopenia management strategy of high-protein intake.

9. Frampton J, Murphy KG, Frost G, Chambers ES. Short-chain fatty acids as potential regulators of skeletal muscle metabolism and function [published online ahead of print, 2020 Mar 30]. Nat Metab 2020; 10.1038/s42255-0200188-7.

10. Sovran $B$, Hugenholtz F, Elderman $M$, et al. Age-associated impairment of the

- mucus barrier function is associated with profound changes in microbiota and immunity. Sci Rep 2019; 9:1437.

This study showed the loss of intestinal barrier function and alteration of fecal microbial composition that occurs with aging, which has been found to be a contributor to loss of muscle quality.

11. Kavanagh $\mathrm{K}$, Hsu FC, Davis AT, et al. Biomarkers of leaky gut are related to

- inflammation and reduced physical function in older adults with cardiometabolic disease and mobility limitations. Geroscience 2019; 41:923-933.

This article connected bacterial translocation with inflammation and muscle function.

12. Margiotta $E$, Miragoli $F$, Callegari $M L$, et al. Gut microbiota composition and frailty in elderly patients with Chronic Kidney Disease. PLoS One 2020; 15:e0228530.

13. Ghetti FF, De Oliveira DG, De Oliveira JM, et al. Effects of dietary intervention on gut microbiota and metabolic-nutritional profile of outpatients with nonalcoholic steatohepatitis: a randomized clinical trial. J Gastrointestin Liver Dis 2019; 28:279-287

14. McGilvray WD, Johnson $B$, Wooten $H$, et al. Immune system stimulation

n. reduces the efficiency of whole-body protein deposition and alters muscle fiber characteristics in growing pigs. Animals (Basel) 2019; 9:323.

This study demonstrated the role of $E$. Coli-induced immune system stimulation on muscle protein metabolism and alteration in fiber histology, which is a direct evidence of microbiome-induced change in muscle physiology and is indeed remarkable.
15. Bjørkhaug ST, Aanes $\mathrm{H}$, Neupane SP, et al. Characterization of gut microbiota composition and functions in patients with chronic alcohol overconsumption. Gut Microbes 2019; 10:663-675.

16. Román E, Nieto JC, Gely $C$, et al. Effect of a multistrain probiotic on cognitive function and risk of falls in patients with cirrhosis: a randomized trial. Hepatol Commun 2019; 3:632-645.

17. Sprooten RTM, Lenaerts $\mathrm{K}$, Braeken DCW, et al. Increased small intestinal permeability during severe acute exacerbations of COPD. Respiration 2018; 95:334-342.

18. Ceelen JJM, Schols AMWJ, Kneppers AEM, et al. Altered protein turnover signaling and myogenesis during impaired recovery of inflammation-induced muscle atrophy in emphysematous mice. Sci Rep 2018; 8:10761

19. Bellikci-Koyu E, Sarer-Yurekli BP, Akyon Y, et al. Effects of regular Kefir consumption on gut microbiota in patients with metabolic syndrome: a parallel-group, randomized, controlled study. Nutrients 2019; 11:2089.

20. Chen $Y$, Feng $R$, Yang $X$, et al. Yogurt improves insulin resistance and liver fat in obese women with nonalcoholic fatty liver disease and metabolic syndrome: a randomized controlled trial. Am J Clin Nutr 2019; 109:1611-1619.

21. Dhakan DB, Maji $A$, Sharma $A K$, et al. The unique composition of Indian gut

- microbiome, gene catalogue, and associated fecal metabolome deciphered using multiomics approaches. GigaScience 2019; 8:.

This study illustrates the distinct composition of Indian gut microbiome, and suggests its possible association with chronic inflammation.

22. Pareek S, Kurakawa T, Das B, et al. Comparison of Japanese and Indian intestinal microbiota shows diet-dependent interaction between bacteria and fungi. NPJ Biofilms Microbiomes 2019; 5:37.

23. Chaudhari DS, Dhotre DP, Agarwal DM, et al. Gut, oral and skin microbiome of Indian patrilineal families reveal perceptible association with age. Sci Rep 2020; 10:5685.

24. Tuikhar N, Keisam S, Labala RK, et al. Comparative analysis of the gut microbiota in centenarians and young adults shows a common signature across genotypically nonrelated populations. Mech Ageing Dev 2019; 179:23-35

25. Zhang S, Chen DC. Facing a new challenge: the adverse effects of antibiotics on gut microbiota and host immunity. Chin Med J (Engl) 2019; 132:1135-1138.

26. Monaghan TM, Sloan TJ, Stockdale SR, et al. Metagenomics reveals impact of

- geography and acute diarrheal disease on the Central Indian human gut microbiome. Gut Microbes 2020; 1-24. [Epub ahead of print]

This study enhances the effect of antibiotic overuse and a high burden of antimicrobial resistance genes in gut microbiome of Indians.

27. Iyer LR, Verma AK, Paul J, Bhattacharya A. Phagocytosis of gut bacteria by

- Entamoeba histolytica. Front Cell Infect Microbiol 2019; 9:34.

This study is especially relevant as it illustrates the loss of beneficial bacterial communities induced by Entamoeba histolytica phagocytosis.

28. Shin JH, Jung S, Kim SA, et al. Differential effects of typical Korean versus American-style diets on gut microbial composition and metabolic profile in healthy overweight Koreans: a randomized crossover trial. Nutrients 2019; $11: 2450$.

29. Singh R, Haque MM, Mande SS. Lifestyle-induced microbial gradients: an Indian perspective. Front Microbiol 2019; 10:2874

This publication highlights the impact of dietary and lifestyle variation on microbial diversity of different Indian communities that may be of great importance.

30. Kaur K, Khatri I, Akhtar A, et al. Metagenomics analysis reveals features unique to Indian distal gut microbiota. PLoS One 2020; 15:e0231197.

31. Shetty SA, Marathe NP, Shouche YS. Opportunities and challenges for gut microbiome studies in the Indian population. Microbiome 2013; 1:24.

32. Kang L, Gao Y, Liu X, et al. Effects of whey protein nutritional supplement on muscle function among community-dwelling frail older people: a multicenter study in China. Arch Gerontol Geriatr 2019; 83:7-12.

33. Liberman $\mathrm{K}, \mathrm{Njemini} \mathrm{R}$, Luiking $\mathrm{Y}$, et al. Thirteen weeks of supplementation of vitamin $\mathrm{D}$ and leucine-enriched whey protein nutritional supplement attenuates chronic low-grade inflammation in sarcopenic older adults: the PROVIDE study. Aging Clin Exp Res 2019; 31:845-854.

34. Huang WC, Lee MC, Lee CC, et al. Effect of Lactobacillus plantarum TWK10

- on exercise physiological adaptation, performance, and body composition in healthy humans. Nutrients 2019;11:2836.

This study directly associated high dose probiotic supplementation with increase in muscle mass.

35. Theou $\mathrm{O}$, Jayanama $\mathrm{K}$, Fernández-Garrido J, et al. Can a prebiotic formulation reduce frailty levels in older people? J Frailty Aging 2019; 8:48-52.

36. Toda $K$, Yamauchi $Y$, Tanaka A, et al. Heat-Killed Bifidobacteriumbreve B-3 enhances muscle functions: possible involvement of increases in muscle mass and mitochondrial biogenesis. Nutrients 2020; 12:219.

37. Chambers ES, Byrne CS, Morrison DJ, et al. Dietary supplementation with inulin-propionate ester or inulin improves insulin sensitivity in adults with overweight and obesity with distinct effects on the gut microbiota, plasma metabolome and systemic inflammatory responses: a randomised cross-over trial. Gut 2019; 68:1430-1438. 